\title{
Estudo comparativo do anel valvar mitral e do ventrículo esquerdo na cardiomiopatia dilatada
}

\author{
Alexandre Ciappina HUEB*, Fabio B. JATENE*, Luiz Felipe P. MOREIRA*, Pablo M. A. POMERANTZEFF*, \\ Bruno Mahler MIOTO*, Renato Coifman CHABELMANN*, Sérgio Almeida de OLIVEIRA*
}

RBCCV 44205-562

Hueb A C, Jatene F B, Moreira L F P, Pomerantzeff P M A, Mioto B M, Chabelmann R C, Oliveira $S A$ - Estudo comparativo do anel valvar mitral e do ventrículo esquerdo na cardiomiopatia dilatada. Rev Bras Cir Cardiovasc 2001; 16(4): 354-63

RESUMO: Objetivo: O objetivo deste trabalho foi analisar o comportamento do anel valvar mitral e do ventrículo esquerdo na cardiomiopatia dilatada (CMD).

Material e Métodos: Analisaram-se 68 corações humanos adultos, fixados, sendo 48 portadores de CMD de etiologia isquêmica ou idiopática, e 20 corações sem cardiopatia. Obteve-se imagens digitalizadas do perímetro do anel mitral, inserção da cúspide anterior e posterior e da porção fibrosa e muscular. Obteve-se também o perímetro interno do VE, distância do septo ao músculo papilar anterior e posterior, distância entre os músculos papilares, e extensão do septo interventricular.

Resultados: A análise dos resultados demonstrou proporcionalidade, tanto da porção fibrosa $\left(r^{2}=\right.$ $0.98)$, quanto da porção muscular $\left(r^{2}=0.99\right)$ do anel em relação ao grau de dilatação da valva mitral. A regressão linear revelou que o perímetro de inserção das cúspides anterior $\left(r^{2}=0.96\right)$ e posterior $\left(r^{2}=0.98\right)$ também guardam relação de proporcionalidade. Observou-se que a dilatação do VE ocorre de forma global e esférica em seus segmentos. Não se observou relação de proporcionalidade entre o grau de dilatação do anel mitral e do VE.

Conclusão: Ao contrário do que se pensava, na CMD de etiologia isquêmica ou idiopática, a dilatação do anel mitral ocorre de forma proporcional e não apenas na sua porção posterior. Não é o grau de dilatação do VE que determina o grau de dilatação do anel mitral pois eles ocorrem de forma independente. Estas observações abrem novas perspectivas para as técnicas de correção da insuficiência valvar mitral na CMD.

DESCRITORES: Valva mitral, patologia. Valva mitral, anatomia \& histologia. Miocardiopatia, complicações Miocardiopatia, patologia. Ventrículo cardíaco, anatomia \& histologia. Ventrículo cardíaco, patologia.

\footnotetext{
Trabalho realizado no Instituto do Coração do Hospital das Clínicas da Faculdade de Medicina da Universidade de São Paulo. São Paulo, SP, Brasil. Apresentado ao $27^{\circ}$ Congresso Nacional de Cirurgia Cardíaca. Rio de Janeiro, RJ, 23 a 25 de março de 2000.

* Do Instituto do Coração do Hospital das Clínicas da Faculdade de Medicina da Universidade de São Paulo.

Endereço para correspondência: Alexandre Ciappina Hueb. Av. Dr. Enéas de Carvalho Aguiar, 44. Divisão de Cirurgia. Cerqueira César. São Paulo, SP, Brasil. CEP: 05403-000. Tel.: (11) 3069-5248. e.mail: hueb@uol.com.br
} 
Hueb A C, Jatene F B, Moreira L F P, Pomerantzeff P M A, Mioto B M, Chabelmann R C, Oliveira S A - Estudo comparativo do anel valvar mitral e do ventrículo esquerdo na cardiomiopatia dilatada. Rev Bras Cir Cardiovasc 2001; 16(4): 354-63

\section{INTRODUÇÃO}

A análise das dimensões do anel valvar mitral na cardiomiopatia dilatada (CMD) tem fundamental importância para o entendimento da gênese da disfunção valvar. Acredita-se que disfunção valvar observada na CMD relacione-se com a dilatação do ventrículo esquerdo (VE) e do átrio esquerdo ( $\mathrm{AE})$, dilatação do anel mitral, distensão das cordas tendíneas, anormalidades na contração dos músculos papilares e da parede do VE (1).

A regurgitação valvar presente nos portadores de CMD isquêmica ou idiopática é fator preditivo de mau prognóstico (2) e uma complicação freqüente na fase final das cardiomiopatias que contribui para agravar a insuficiência cardíaca, levando a uma evolução desfavorável (3). A introdução da ecocardiografia foi oportuna e acrescentou dados importantes para a compreensão dos mecanismos responsáveis pela regurgitação valvar mitral observada na CMD, mas, de outra forma, limitou os estudos anatômicos, tão necessários para dar suporte às observações obtidas $(4,5)$.

Análise ecocardiográfica em portadores de CMD, com e sem regurgitação mitral, demonstra que a dilatação do anel mitral ocorre apenas em alguns pacientes e não guarda proporção com o grau de dilatação do VE, sugerindo que a regurgitação valvar que ocorre em associação com a dilatação do VE tenha mecanismo independente da dilatação do anel mitral, como a perda da ação esfincteriana do anel ou o mau alinhamento dos músculos papilares ${ }^{(6)}$.

Estudos anatômicos do anel mitral em corações normais e com CMD demonstraram que a simples dilatação mitral não costuma ser responsável pela regurgitação valvar, sendo necessária uma deformação no esqueleto fibroso, para dilatar o anel a ponto de causar regurgitação valvar (7). Observações em modelos experimentais de VE, demonstram que a regurgitação mitral só ocorre quando a dilatação do anel é superior a 1,75 vezes o tamanho normal, ou 1,50 vezes o tamanho normal, quando há deslocamento apical do músculo papilar pósterolateral, demonstrando que a valva mitral compensa a dilatação anular, devido à ampla superfície de suas cúspides ${ }^{(8)}$.

O conhecimento das alterações do aparelho valvar mitral tem aplicabilidade direta no aprimoramento das diversas técnicas de correção cirúrgica que intervêm sobre o anel, as cúspides, as cordas tendíneas e os músculos papilares, em associações ou isoladamente, justificando seu estudo anatômico na cardiomiopatia dilatada $(9,10)$.

\section{MATERIAL E METÓDOS}

Sessenta e oito corações humanos adultos, fixados em formaldeído a10\%, em bom estado de conservação, foram assim agrupados: GRUPO I: 48 corações com CMD de etiologia isquêmica ou idiopática, dos quais $43(89,5 \%)$ pertenciam a indivíduos do sexo masculino. A idade variou de 19 a 79 anos, com média de 56,8 anos e peso de 400 a 1200 gramas, com média de 743,8 gramas; GRUPO II: 20 corações sem cardiopatia, onde 18 (90\%) eram do sexo masculino. A idade variou de 19 a 56 anos, com média de 32,6 anos e peso de 203 a 313 gramas, com média de 257,3 gramas.

GRUPO I com 48 corações foi subdividido em:

- CMD isquêmica: 24 corações com CMD de etiologia isquêmica;

- CMD idiopática: 24 corações com CMD de etiologia idiopática.

O GRUPO II com 20 corações de cadáveres humanos, fixados, oriundos de cadáveres sem cardiopatia prévia cuja "causa mortis" foi o trauma. Para caracterizar os corações com CMD utilizaramse critérios $(10,11)$ :

- Diâmetro medido na borda interna do VE, ao nível da meia altura do corpo do músculo papilar anterior, valor igual ou superior a $5,5 \mathrm{~cm}$;

- Perímetro medido na borda interna do VE, ao nível da meia altura do corpo do músculo papilar anterior, valor igual ou superior a $15,0 \mathrm{~cm}$.

Os critérios de exclusão para CMD de etiologia idiopática foram: idade inferior a 18 anos; doença isquêmica coronariana; anomalias cardíacas congênitas; anomalias cardíacas valvares; anomalias no septo interventricular, interatrial ou patência do forame oval; doença miocárdica infiltrativa, como amiloidose; bloqueio atrioventricular total; doença hipertensiva com níveis sistólicos acima de 200 $\mathrm{mmHg}$; relação com o período puerperal; prova de Machado-Guerreiro positiva; presença de deformidades da cavidade do VE; tratamento cirúrgico das cavidades ventriculares e/ou das valvas atrioventriculares ou semilunares.

Utilizaram-se os mesmos critérios de exclusão de CMD idiopática para caracterizar os corações com CMD de etiologia isquêmica, com exceção, da presença de doença isquêmica do coração. Neste caso, o critério de inclusão para CMD isquêmica foi a presença de lesão coronariana superior a $70 \%$ do lúmen vascular, em uma ou mais artérias coronárias.

Estabeleceram-se, como critérios de inclusão dos vinte corações obtidos de cadáveres sem 
Hueb A C, Jatene F B, Moreira L F P, Pomerantzeff P M A, Mioto B M, Chabelmann R C, Oliveira S A - Estudo comparativo do anel valvar mitral e do ventrículo esquerdo na cardiomiopatia dilatada. Rev Bras Cir Cardiovasc 2001; 16(4): 354-63

cardiopatia prévia: ausência de alterações cardíacas macroscópicas, particularmente de lesões valvares; idade superior a 18 anos e inferior a 70 anos.

\section{Método para Preparo e Fixação dos Corações}

Após seleção, procedeu-se a secção dos grandes vasos ao nível das comissuras valvares, das veias cavas e pulmonares em sua junção com os átrios, secção do átrio direito e esquerdo, ao nível da junção atrioventricular, permitindo visibilização das valvas atrioventriculares esquerda e direita, e secção transversal das paredes dos ventrículos esquerdo e direito ao nível da meia altura do corpo do músculo papilar anterior (Figura 1).

\section{Método para Obtenção das Medidas}

Obtiveram-se medidas através de fotografias digitalizadas que foram analisadas pelo computa- dor. Para mensuração dos perímetro do anel mitral e da cavidade ventricular, as imagens dos corações foram submetidas a programa de "software" para a obtenção de resultados mais fidedignos e passíveis de reprodução.

Sob uma mesa com suporte para máquina fotográfica digital, foram posicionados os corações, utilizado uma régua milimetrada para calibração da imagem e obtenção das imagens das valvas atrioventriculares e das cavidades ventriculares. Essas imagens foram digitalizadas e, através de programa de software, analisaramse as distâncias e perímetros da valva mitral e da cavidade do VE.

$\mathrm{Na}$ cavidade ventricular esquerda, analisou-se: (1) Distância do Septo Interventricular ao Músculo Papilar Posterior; (2) Distância entre os Músculos Papilares; (3) Distância do Septo Interventricular ao Músculo Papilar Anterior; (4) Extensão do Septo Interventricular e o Perímetro Interno do VE, através da soma dessas variáveis (Figura 2).
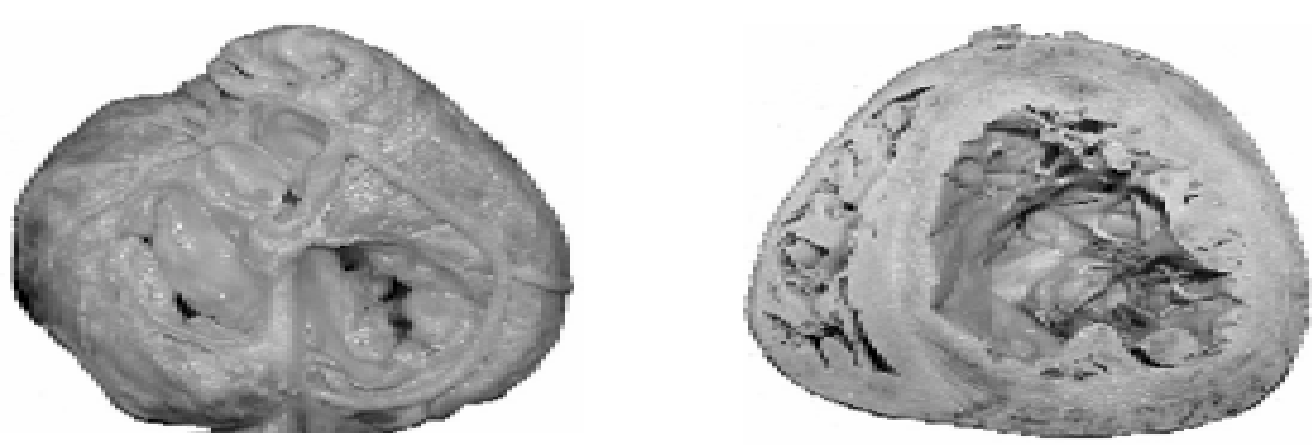

Fig. 1 - Coração com CMD após rebatidas as cavidades atriais expondo as valvas atrioventriculares esquerda e direita (esquerda). Coração com CMD seccionado na $1 / 2$ altura do músculo papilar anterior, onde se observam as cavidades ventriculares esquerda e direita (direita).

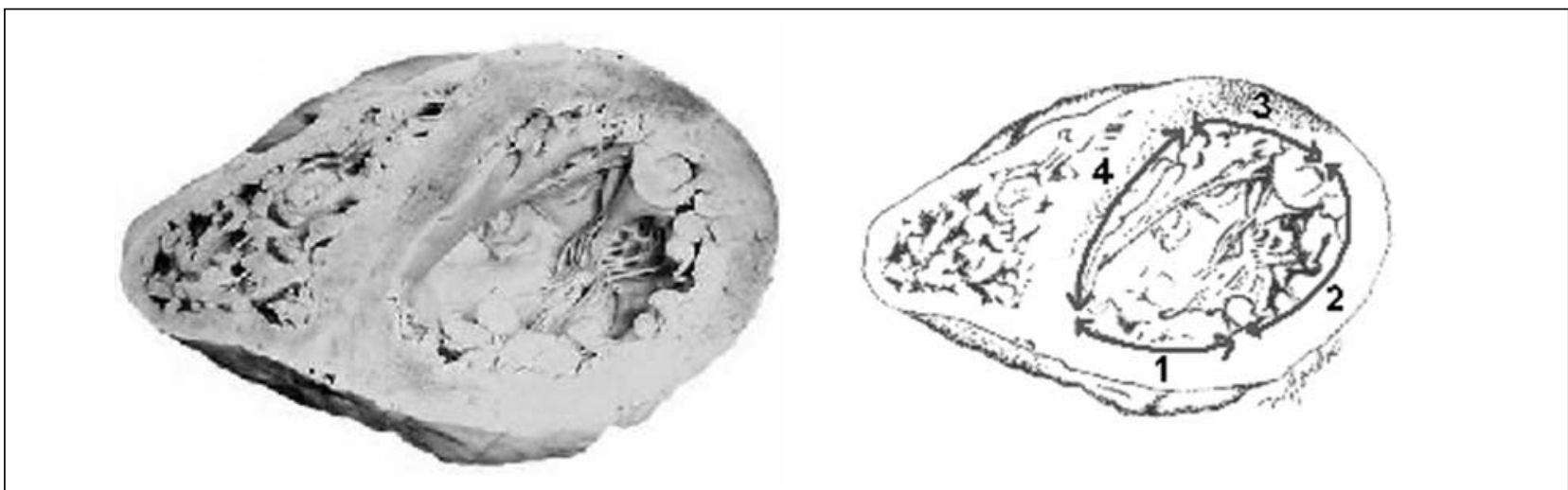

Fig. 2 - Cavidades ventriculares seccionadas na $1 / 2$ altura do músculo papilar anterior, demonstrando as variáveis: (1) Distância do Septo Interventricular ao Músculo Papilar Posterior, (2) Distância entre os Músculos Papilares, (3) Distância do Septo Interventricular ao Músculo Papilar Anterior, (4) Extensão do Septo Interventricular e o Perímetro Interno do VE pela soma das medidas. 
Hueb A C, Jatene F B, Moreira L F P, Pomerantzeff P M A, Mioto B M, Chabelmann R C, Oliveira S A - Estudo comparativo do anel valvar mitral e do ventrículo esquerdo na cardiomiopatia dilatada. Rev Bras Cir Cardiovasc 2001; 16(4): 354-63

No anel valvar mitral analisou-se: (A) Perímetro de Inserção da Cúspide Anterior da Valva Mitral; (B) Perímetro de Inserção da Cúspide Posterior da Valva Mitral; (C) Menor Distância Perimetral entre os Trígonos Fibrosos (porção fibrosa); (D) Maior Distância Perimetral entre os Trígonos Fibrosos (porção muscular) e o Perímetro do Anel Valvar Mitral através da soma dessas variáveis (Figura 3).

\section{Análise Estatística}

Os diversos parâmetros analisados foram comparados através da análise de Variância de um Fator, sendo discriminadas as diferenças entre os grupos pelo teste $t$ de Bouferrani.

A existência de correlação entre variáveis foi analisada através de regressão linear.

Os dados foram apresentados em média com o desvio padrão. Foi estabelecido o nível de significância de $5 \%$.

\section{RESULTADOS}

As variáveis obtidas nos 3 grupos: corações normais, corações com CMD isquêmica e corações com CMD idiopática, foram dispostas da seguinte forma:

A - Análise das Variáveis do Anel Mitral e do Ventrículo Esquerdo;

B - Comparação entre o Perímetro do Anel Mitral e o Perímetro do VE nos Corações com CMD Isquêmica e Idiopática;

C - Comparação entre as Variáveis do Anel Mitral em Corações Normais e com CMD.

\section{A - Análise das Variáveis do Anel Mitral e do Ventrículo Esquerdo}

Quando foram comparados o grupo de corações normais com o de corações com CMD, os resultados mostraram que houve aumento do perímetro da valva mitral, das distâncias perimetrais entre os trígonos fibrosos e do perímetro de inserção da cúspide posterior da valva mitral com $p=0.0001$ (Tabela 1).

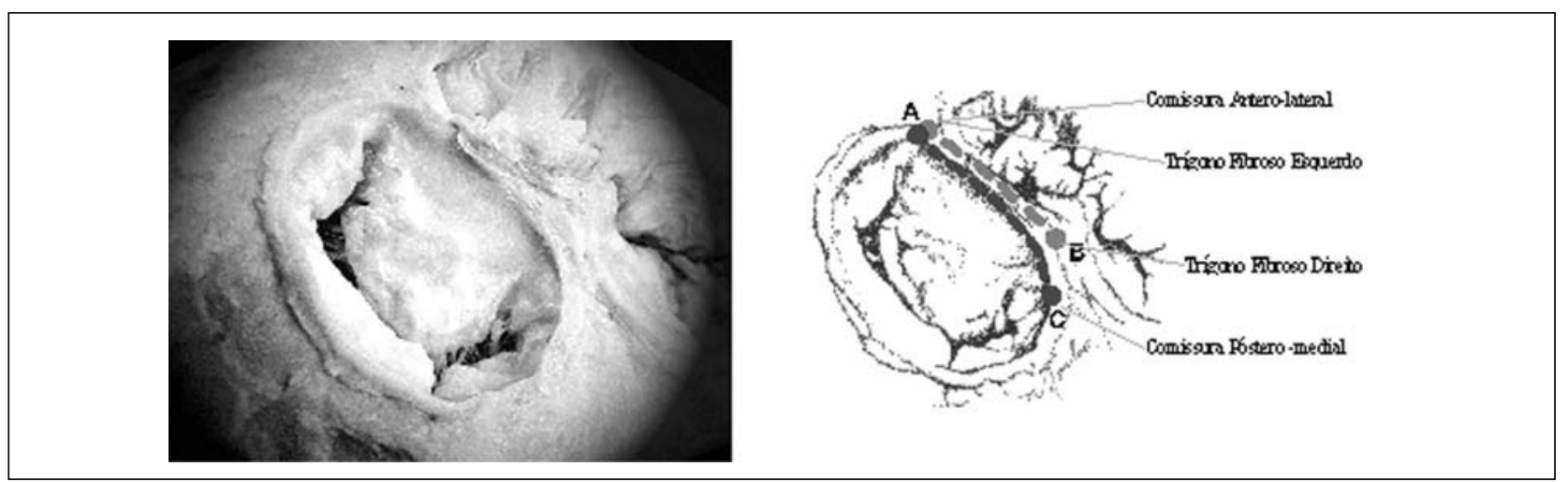

Fig. 3 - Base do coração e valva mitral, demonstrando as variáveis: (A-B) Menor Distância Perimetral entre os Trígonos Fibrosos (porção fibrosa); (B-A) Maior Distância Perimetral entre os Trígonos Fibrosos (porção muscular); (A-C) Perímetro de Inserção da Cúspide Anterior da Valva Mitral; (C-A) Perímetro de Inserção da Cúspide Posterior da Valva Mitral.

TABELA 1

\begin{tabular}{|c|c|c|c|c|}
\hline \multicolumn{5}{|c|}{ MEDIDAS RELACIONADAS AO ANEL MITRAL NOS CORAÇÕES NORMAIS E COM CMD ISQUÊMICA E IDIOPÁTICA* } \\
\hline Variável & $\begin{array}{l}\text { Corações } \\
\text { Normais } \\
20 \text { Peças }\end{array}$ & $\begin{array}{c}\text { CMD } \\
\text { Isquêmica } \\
24 \text { Peças }\end{array}$ & $\begin{array}{c}\text { CMD } \\
\text { Idiopática } \\
24 \text { Peças }\end{array}$ & $p$ \\
\hline Per Mi & $8.80 \pm 0.87$ & $10.30 \pm 1.05$ & $11.28 \pm 1.28$ & $p=0.0001$ \\
\hline Trig ED & $1.90 \pm 0.24$ & $2.62 \pm 0.32$ & $2.74 \pm 0.42$ & $p=0.0001$ \\
\hline Trig DE & $6.90 \pm 0.75$ & $7.68 \pm 0.89$ & $8.54 \pm 1.05$ & $p=0.0001$ \\
\hline Cusp Ant & $3.79 \pm 0.67$ & $4.06 \pm 0.89$ & $4.56 \pm 0.86$ & $p=0.05$ \\
\hline Cusp Post & $5.01 \pm 0.85$ & $6.24 \pm 1.11$ & $6.72 \pm 1.38$ & $p=0.0001$ \\
\hline
\end{tabular}

Per Mi: perímetro do anel mitral, Trig ED: menor distância perimetral entre os trígonos fibrosos, Trig DE: maior distância perimetral entre os trígonos fibrosos, Cusp Ant: perímetro de inserção da cúspide anterior da valva mitral, Cusp Post: perímetro de inserção da cúspide posterior da valva mitral. * Média em centímetros com desvio padrão. 
Hueb A C, Jatene F B, Moreira L F P, Pomerantzeff P M A, Mioto B M, Chabelmann R C, Oliveira S A - Estudo comparativo do anel valvar mitral e do ventrículo esquerdo na cardiomiopatia dilatada. Rev Bras Cir Cardiovasc 2001; 16(4): 354-63

Observou-se que as medidas obtidas nos corações com CMD idiopática têm valores superiores às medidas obtidas dos corações com CMD isquêmica.

A Tabela 2 mostra os valores de $p$ quando comparados o grupo de CMD isquêmica, CMD idiopática e corações normais, demonstrando a significância entre os grupos.

Observa-se no Gráfico 1 o crescimento proporcional da porção fibrosa em relação à porção muscular do anel mitral.

A análise das variáveis do ventrículo esquerdo, entre elas: perímetro do VE, distância do músculo papilar ao septo, distância interpapilar, distância do músculo papilar anterior ao septo e extensão do septo nos corações normais em relação aos corações com CMD idiopática e isquêmica revelou que o crescimento das diferentes variáveis tem comportamento semelhante, ou seja, o crescimento é global e esférico (Gráfico 2).

Observou-se que as medidas obtidas nos corações com CMD idiopática têm valores superiores às medidas obtidas dos corações com CMD isquêmica.
GRÁFICO 1

PORÇÃO FIBROSA E MUSCULAR DO ANEL MITRAL EM CENTÍMETROS

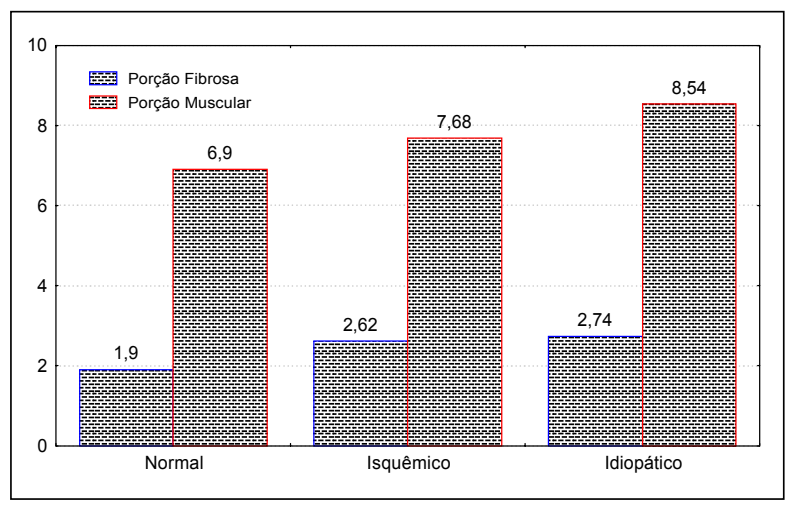

Quando comparados os corações normais com corações com cardiomiopatia, observou-se diferença estatística que todas as variáveis com $p<0,05$.

B - Comparação entre o Perímetro do Anel Mitral e o Perímetro do VE nos Corações com CMD Isquêmica e Idiopática

TABELA 2

\begin{tabular}{lccc}
\hline \multicolumn{2}{c}{ ANÁLISE DAS VARIÁVEIS DO ANEL MITRAL QUANDO COMPARADOS OS CORAÇÕES NORMAIS E COM CMD* } \\
\hline Variável & $\begin{array}{c}\text { CMD isquêmica } \\
\text { CMD Idiopática }\end{array}$ & CMD Isquêmica & C \\
& $p<0.05$ & Normal & CMD Idiopática \\
X & Normal \\
\hline Per Mi & NS & $p<0.05$ & $p<0.05$ \\
Trig ED & $p<0.05$ & $p<0.05$ & $p<0.05$ \\
Trig DE & NS & $p<0.05$ & $p<0.05$ \\
Cusp Ant & NS & NS & $p<0.05$ \\
Cusp Post & & $p<0.05$ & $p<0.05$ \\
\hline
\end{tabular}

Per Mi: perímetro do anel mitral, Trig ED: menor distância perimetral entre os trígonos fibrosos, Trig DE: maior distância perimetral entre os trígonos fibrosos, Cusp Ant: perímetro de inserção da cúspide anterior da valva mitral, Cusp Post: perímetro de inserção da cúspide posterior da valva mitral. * Média em centímetros com desvio padrão.

TABELA 3

\begin{tabular}{|c|c|c|c|c|}
\hline \multicolumn{5}{|c|}{ VARIÁVEIS DO VE NOS CORAÇÕES NORMAIS E COM CMD ISQUÊMICA E IDIOPÁTICA* } \\
\hline Variável & $\begin{array}{l}\text { Corações } \\
\text { Normais } \\
20 \text { Peças }\end{array}$ & $\begin{array}{l}\text { CMD } \\
\text { Isquêmica } \\
\text { 24 Peças }\end{array}$ & $\begin{array}{c}\text { CMD } \\
\text { Idiopática } \\
24 \text { Peças }\end{array}$ & $p$ \\
\hline Per VE & $13.26 \pm 1.83$ & $19.16 \pm 1.84$ & $21.28 \pm 1.84$ & $p=0.0001$ \\
\hline Pap Post & $1.88 \pm 0.45$ & $3.49 \pm 0.72$ & $3.96 \pm 0.90$ & $p=0.0001$ \\
\hline Dinter & $3.20 \pm 0.70$ & $4.76 \pm 0.79$ & $5.40 \pm 0.80$ & $p=0.0001$ \\
\hline Pap Ant & $3.26 \pm 0.89$ & $4.80 \pm 1.19$ & $5.35 \pm 1.04$ & $p=0.0001$ \\
\hline Septo & $4.92 \pm 0.78$ & $6.11 \pm 1.13$ & $6.56 \pm 0.85$ & $p=0.0001$ \\
\hline
\end{tabular}

Per VE: perímetro do ventrículo esquerdo, Pap Post: distância do septo interventricular ao músculo papilar posterior, Dinter: distância entre o músculo papilar anterior e posterior, Pap Ant: distância do septo interventricular ao músculo papilar anterior, Septo: extensão do septo interventricular. * Média em centímetros com desvio padrão. 
Hueb A C, Jatene F B, Moreira L F P, Pomerantzeff P M A, Mioto B M, Chabelmann R C, Oliveira S A - Estudo comparativo do anel valvar mitral e do ventrículo esquerdo na cardiomiopatia dilatada. Rev Bras Cir Cardiovasc 2001; 16(4): 354-63

Observou-se aumento progressivo na variável perímetro do VE e perímetro da valva mitral quando se compararam corações normais com corações com CMD isquêmica e idiopática, respectivamente. Submetidos a regressão linear, observou-se que tanto na CMD isquêmica, quanto na CMD idiopática, o perímetro do VE não guarda proporção de linearidade com o perímetro da valva mitral, ou seja, o grau de dilatação do VE não guarda relação com o grau de dilatação do anel mitral (Gráfico 3).

\section{C - Comparação entre as Variáveis do Anel Mitral em Corações Normais e com CMD}

Analisando conjuntamente as variáveis do anel mitral, nos corações normais e com CMD e, plotandoos para um gráfico de regressão linear, observouse que as distâncias entre os trígonos fibrosos guardam relação de proporcionalidade com o grau de dilatação do anel mitral, tanto para a porção fibrosa $\left(r^{2}=0.98\right)$, quanto para a porção muscular do anel $\left(r^{2}=0.99\right)$. Vale dizer, em decorrência da significância, que, proporcionalmente o aumento das distâncias entre os trígonos fibrosos é semelhante (Gráfico 4).

Observou-se que a porção fibrosa ocupa $21.5 \%$ do perímetro do anel nos corações normais e $24.8 \%$ nos corações com CMD. O perímetro de inserção da cúspide anterior ocupa $43 \%$ do anel nos corações normais e $39 \%$ nos corações com CMD.

GRÁFICO 2

PORCENTAGEM DE OCUPAÇÃO DAS VARIÁVEIS ANALISADAS NO VE EM CORAÇÕES NORMAIS E COM CMD

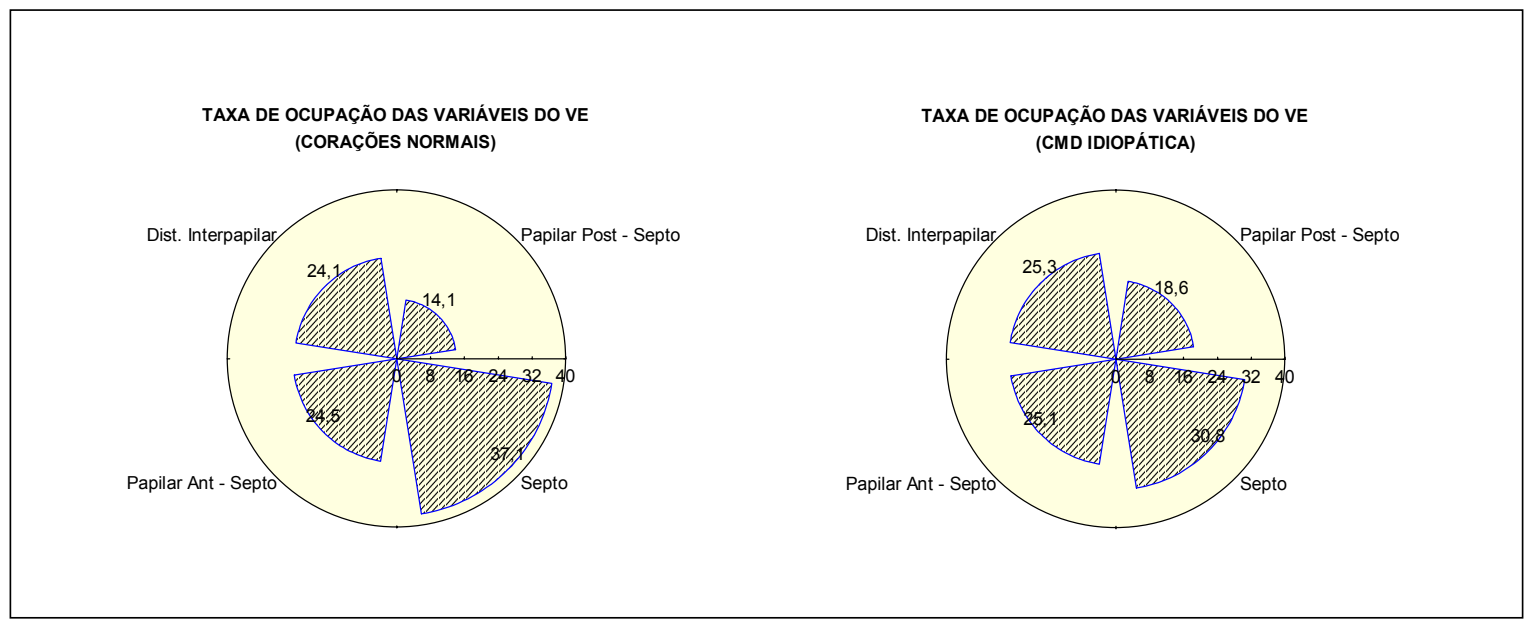

GRÁFICO 3

PERÍMETRO DO VE E PERÍMETRO DO ANEL MITRAL NAS CMD ISQUÊMICA E IDIOPÁTICA (EM CENTÍMETROS)

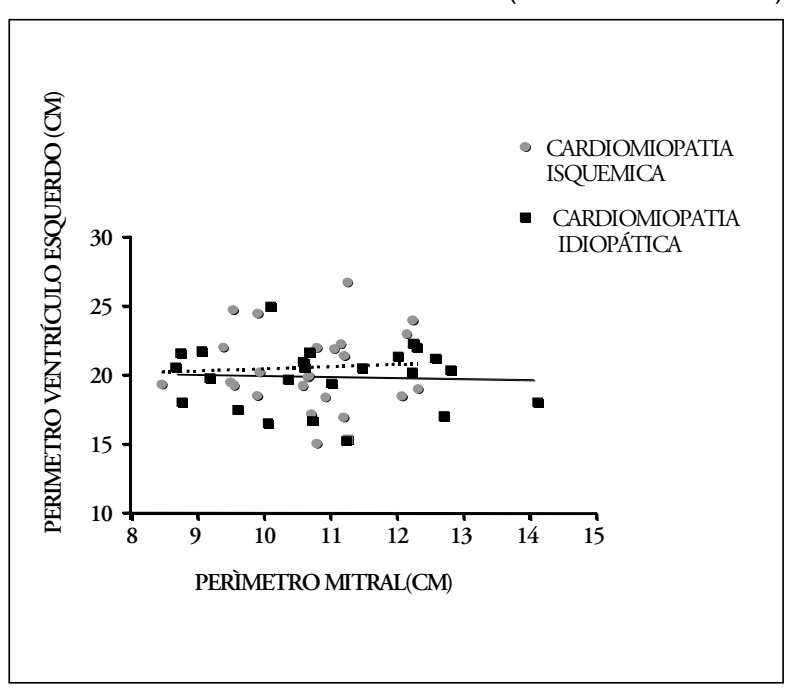

GRÁFICO 4

PORÇÃO FIBROSA E MUSCULAR EM RELAÇÃO AO PERIIMETRO DO ANEL MITRAL (EM CENTÍMETROS)

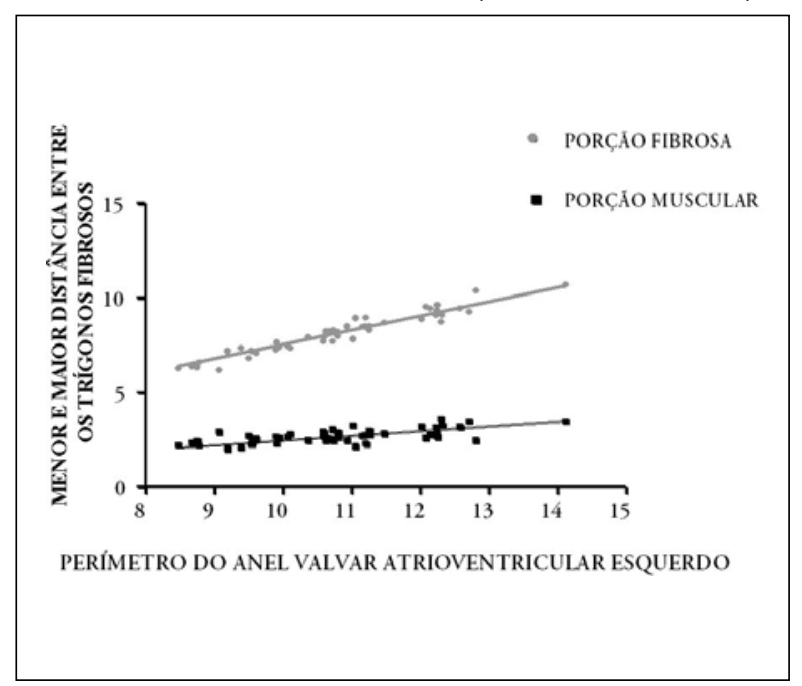


Pelo fato do perímetro de inserção da cúspide anterior e posterior do anel mitral ocupar o perímetro do anel de forma mais homogênea, essas variáveis também foram analisadas em relação ao perímetro da valva.

A regressão linear revelou que o perímetro de inserção das cúspides anterior $\left(r^{2}=0.96\right)$ e posterior $\left(r^{2}=0.98\right)$ também guardam relação de proporcionalidade quando relacionados à dilatação do anel valvar mitral (Gráfico 5).

GRÁFICO 5

PERÍMETRO DE INSERÇÃO DAS CÚSPIDES ANTERIOR

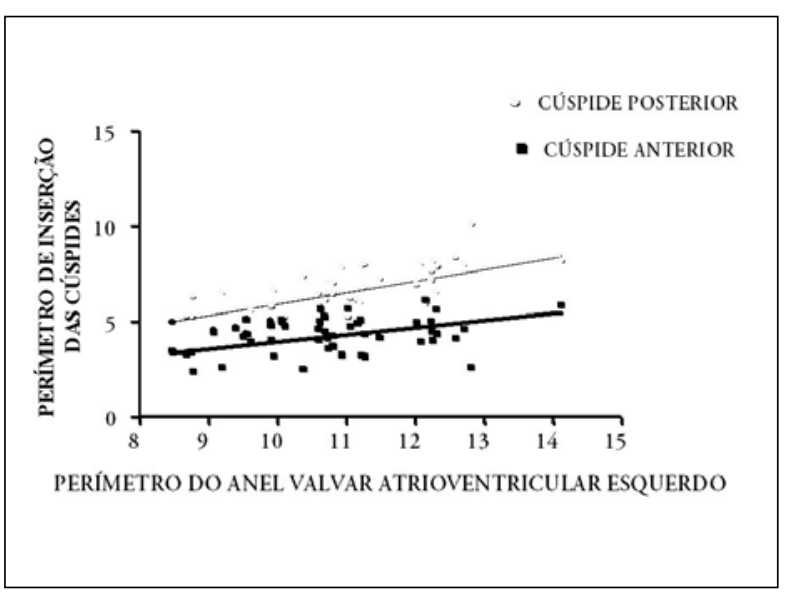

\section{COMENTÁRIOS}

O mecanismo de fechamento da valva atrioventricular esquerda não está totalmente esclarecido. O anel, considerado inerte por muito tempo, não somente serve de sustentação para inserção das cúspides, como também, durante a sístole, diminui sua circunferência, reduzindo a área do orifício valvar a ser fechado (13).

A insuficiência valvar mitral é comum em pacientes portadores de CMD isquêmica ou idiopática e, embora o verdadeiro mecanismo da insuficiência funcional não esteja ainda totalmente esclarecido, admite-se concomitância de fatores como o deslocamento dos músculos papilares, o tracionamento das inserções valvares junto ao anel fibroso, e a diminuição da força contrátil do ventrículo esquerdo arrefecendo a pressão transvalvar como prováveis fatores etiológicos (14).

Estudos anatômicos (7) demonstraram que a dilatação do anel valvar mitral é uma causa rara de regurgitação, sendo necessário uma anormalidade no esqueleto fibroso do coração para que o anel dilate o bastante a ponto de causar regurgitação mitral.
O anel valvar mitral é composto de duas estruturas fibrosas situadas anteriormente, os trígonos fibrosos direito e esquerdo, mas seu segmento posterior é desprovido de estruturas fibrosas que, teoricamente, poderiam sofrer dilatação.

GLASSON et al. (15), utilizando marcadores radiopacos e videofluoroscopia biplana, descreveram que o anel valvar mitral é uma estrutura dinâmica, que sofre alterações na forma e no tamanho de todos os segmentos do anel, tanto da porção posterior quanto da anterior; demonstrando que a dinâmica da cúspide anterior pode ser um componente muito mais ativo no aparato valvar atrioventricular esquerdo do que previamente se pensava.

Em nosso estudo, a análise das valvas "in situ" com utilização de programas de software possibilitou a avaliação dos perímetros de inserção das cúspides e das distâncias entre os trígonos fibrosos do anel mitral. A análise desses perímetros com a valva excisada pode comprometer sua avaliação. KUNZELMAN et al. (16), comparando o perímetro valvar mitral e a extensão da inserção de cada cúspide "in situ" e após excisão da valva, observaram aumento de $31 \%$ na inserção da cúspide posterior e $3,3 \%$ da cúspide anterior quando as mesmas foram excisadas.

Frente a uma gama de possibilidades, optou-se pela análise de cinco variáveis no anel valvar mitral, para verificar seu comportamento na CMD isquêmica e idiopática: perímetro, porção fibrosa e muscular do anel, perímetro de inserção da cúspide anterior e posterior.

Segundo vários autores $(8,13,17,18)$, a menor distância perimetral entre os trígonos fibrosos, por pertencer ao esqueleto fibroso do coração, é uma área indistensível.

O nosso estudo comparou corações normais com corações com CMD, e demonstrou que houve um aumento proporcional da porção fibrosa e da porção muscular do anel, quando comparados ao grau de dilatação do anel mitral.

Devido ao fato da porção fibrosa ocupar apenas $21,5 \%$ da circunferência mitral, obtivemos também o perímetro de inserção da cúspide anterior, este com $43 \%$ de ocupação da circunferência e ambas as medidas, tanto a menor distância perimetral entre os trígonos fibrosos, que representa a porção fibrosa do anel, como o perímetro de inserção da cúspide anterior, mostraram crescimento proporcional em relação ao crescimento do anel valvar $\left(r^{2} 0,99\right.$ e 0,98 , respectivamente). 
Hueb A C, Jatene F B, Moreira L F P, Pomerantzeff P M A, Mioto B M, Chabelmann R C, Oliveira S A - Estudo comparativo do anel valvar mitral e do ventrículo esquerdo na cardiomiopatia dilatada. Rev Bras Cir Cardiovasc 2001; 16(4): 354-63

Esses dados são conflitantes com os dados da literatura, que desconsideram o crescimento da porção fibrosa do anel $(1,14,19)$ e daqueles autores $(7,20,21)$ que admitem um crescimento mínimo.

Com base nesses dados, surge uma questão. Já que, anatomicamente, a dilatação do anel mitral é proporcional, qual seria o substrato teórico para a realização da anuloplastia assimétrica do anel?

Segundo a literatura (19), estudos anatômicos têm demonstrado que, quando a dilatação do anel ocorre, a distância entre os trígonos fibrosos anteriormente permanece estável e a dilatação ocorre ao longo da inserção da cúspide posterior.

Com base nessa premissa, vários autores (1921) utilizam, como técnica cirúrgica para correção da regurgitação valvar mitral, um anel metálico ou de "teflon", tira de pericárdio autólogo ou a sutura simples para promover um mesmo fenômeno, qual seja, a aproximação da parede posterior do ventrículo esquerdo, representada pela cúspide posterior da valva mitral, em direção à cúspide anterior.

CARPENTIER (22) acreditava que a anuloplastia assimétrica dividia desigualmente as forças sobre o anel valvar atrioventricular esquerdo opondo-se à persistência do processo de dilatação do anel e propôs o remodelamento com uma prótese rígida de "teflon".

DURAN \& UBAGO (23) desenvolveram um anel protético totalmente flexível para reconstrução do anel valvar atrioventricular esquerdo, restaurando o anel ao seu tamanho e forma normais, respeitando as mudanças do anel valvar durante o ciclo cardíaco.

As variáveis ventriculares analisadas demonstram uma dilatação global e proporcional entres os segmentos observados. Segundo KONO et al. (24), as mudanças na cavidade ventricular esquerda que ocorrem durante o curso da insuficiência cardíaca manifestam-se através do aumento da esfericidade da câmara, ou seja, não ocorrem em segmentos e sim globalmente.

As observações demonstraram ausência de correlação entre o grau de dilatação do VE e o grau de dilatação do anel mitral. Esse dado indaga para o fato de qual é o verdadeiro fator determinante para a insuficiência mitral já que se observou que cavidades tão grandes não levam a dilatações anulares tão importantes. Assim como anéis podem estar bastante dilatados com cavidades ventriculares não tão dilatadas.

\section{CONSIDERAÇÕES FINAIS}

Segundo as estatísticas norte americanas, estima-se um aumento anual de 400.000 pacientes em classe funcional IV da NYHA, para ICC, que geram, em média, duas internações hospitalares/ano.

O aparecimento de insuficiência valvar mitral moderada à importante, na fase avançada da ICC, agrava a disfunção ventricular e perpetua a insuficiência cardíaca (25).

Desde 1971, CARPENTIER et al. (26) têm publicado numerosos estudos propondo novas técnicas de reconstrução da valva atrioventricular esquerda. Para a anuloplastia, o anel de Carpentier foi considerado o "gold standard" por muitos anos.

Recentemente, vários autores $(19,27)$ têm demonstrado desvantagens com o uso do anel rígido, incluindo deformação da geometria natural do anel e possível obstrução da via de saída do VE. Conseqüentemente, diferentes propostas surgem para os vários tipos de técnicas de remodelamento, baseadas na redução ou reforço apenas da porção posterior do anel.

Presume-se que a continuidade da porção anterior do anel valvar mitral e o septo interventricular, na sua porção fibrosa, seja a razão anatômica pela qual essa porção não participa da dilatação anular e sustenta as bases da escolha para a realização da anuloplastia envolvendo exclusivamente a porção posterior (28).

Nosso estudo anatômico demonstra que nos corações com CMD de etiologia isquêmica ou idiopática ocorre a dilatação do anel mitral e que esta se faz de forma proporcional tanto da sua porção fibrosa, quanto da muscular, assim como o perímetro de inserção da cúspide anterior e posterior. Ou seja, obtivemos substrato anatômico para justificar não somente a plicatura posterior do anel, mas também a anterior. 
Hueb A C, Jatene F B, Moreira L F P, Pomerantzeff P M A, Oliveira S A - Comparative study of the mitral valve anulus and of the left ventricle in dilated cardiomyopathy. Rev Bras Cir Cardiovasc 2001; 16(4): 354-63

ABSTRACT: Objective: The aim of this investigation was to analyze the behavior of the mitral valve anulus and of the left ventricle (LV) in dilated cardiomyopathy (DCM).

Material and Methods: Sixty-eight hearts of adults males suffering from ischemic or idiopathic DCM were analyzed after formaldehyde fixation and 20 hearts from in former patients without cardiopathy. Images were obtained after digitalization of the perimeter of mitral anulus, insertion of the anterior and posterior cusps and of the fibrous and muscular portion, as well as of the internal perimeter of the LV, distance from the septum to the anterior and posterior papillary muscle and of the septum interventricular length.

Results: The analysis of the results showed proportionality, both of the fibrous portion $\left(r^{2}=0.98\right)$ and of the muscular portion $\left(r^{2}=0.99\right)$ of the anulus as related to the dilatation degree of the mitral valve. Linear regression revealed a proportion relationship between the perimeter of the anterior $\left(r^{2}=\right.$ $0.96)$ and posterior $\left(r^{2}=0.99\right)$ cusps. The LV dilation occurred in global and spherical shape in all segments. No proportional relationship was observed between the degree of dilation of the mitral anulus and of the LV.

Conclusions: Contrary to previous thinking, the DCM of ischemic or idiopathic etiology, the dilation of the anulus occurs in a proportional form and not only in its posterior portion. The degree of LV dilation does not determine the degree of mitral anulus dilation as they occur independently. Such obeservations open new perspectives for the techniques of correction of mitral valve insuficiency in DMC.

DESCRIPTORS: Mitral valve, pathology. Mitral valve, anatomy \& histology. Cardiomyopathy, complications. Cardiomyopathy, pathology. Heart ventricle, anatomy \& histology. Heart ventricle, pathology.

\section{REFERÊNCIAS BIBLIOGRÁFICAS}

1 Oki T, Fukuda N, luchi $A$ et al. - Possible mechanisms of mitral regurgitation in dilated hearts: a study using transesophageal echocardiography. Clin. Cardiol 1996; 19: 639-43.

2 Comin J, Manito N, Roca J, Castells E, Esplingas E Functional mitral regurgitation: physiopathology and impact of medical therapy and surgical techniques for left ventricle reduction. Rev Esp Cardiol 1999; 52: 512-20.

3 Bolling S F, Pagani F D, Deeb G M, Bach D S Intermediate-term outcome of mitral reconstruction in cardiomyopathy. J Thorac Cardiovasc Surg 1998; 115: $381-8$.

4 Otsuji Y, Handschumacher MD, Schwamenthal E D et al. - Insights from three-dimensional echocardiography into the mechanism of functional mitral regurgitation: direct in vivo demonstration of altered leaflet tethering geometry. Circulation 1997; 96: 1999-2008.

5 Walmsley R - Anatomy of human mitral valve in adult cadaver and comparative anatomy of the valve. $\mathrm{Br}$ Heart J 1978; 40: 351-66.

6 Chandraratna P A \& Aranow W S - Mitral valve ring in normal vs dilated left ventricle: cross-sectional echocardiographic study. Chest 1981; 79: 152-4.
7 Bulkley B H \& Roberts W C - Dilatation of the mitral anulus: a rare cause of mitral regurgitation. $A m \mathrm{~J}$ Med 1975; 59: 457-63.

8 He S, Lemmon-Jr. J D, Weston M W, Jensen M O, Levine R A, Yoganathan A P - Mitral valve compensation for annular dilatation: in vitro study into the mechanisms of functional mitral regurgitation with an adjustable annulus model. $J$ Heart Valve Dis 1999; 8: 294-302.

9 Braile D M, Ardito R V, Pinto G H et al. - Plástica mitral. Rev Bras Cir Cardiovasc 1990; 5: 86-98.

10 Kasper E K, Agema W R, Hutchins G M, Deckers J W, Hare J M, Baughman K L - The causes of dilated cardiomyopathy: a clinicopathologic review of 673 consecutive patients. J Am Coll Cardiol 1994; 23: 586-90.

11 Simão Filho C - Remodelamento ventricular esquerdo em cardiomiopatias de diferentes etiologias na sua forma dilatada: estudo morfológico comparativo em peças anatômicas. [Tese. Doutorado] São Paulo: Faculdade de Medicina da Universidade de São Paulo, 1998. 119p.

12 Demaria R, Godlewski G, De Guilhermier P, Tang J, Seguin J, Chaptal P A - Static morphometric bases for CT identification and evaluation of the outflow 
Hueb A C, Jatene F B, Moreira L F P, Pomerantzeff P M A, Mioto B M, Chabelmann R C, Oliveira S A - Estudo comparativo do anel valvar mitral e do ventrículo esquerdo na cardiomiopatia dilatada. Rev Bras Cir Cardiovasc 2001; 16(4): 354-63

chamber of the left ventricle: preliminary study in formalin-fixed heart. Surg Radio. Anat 1993; 15: $145-50$.

13 Perloff J K \& Roberts W C - The mitral apparatus: functional anatomy of mitral regurgitation. Circulation 1972; 46: 227-39.

14 He S, Fontaine A A, Schwammenthal E, Levine R, Yoganathan A P, Levine R A - Integrated mechanism for functional mitral regurgitation: leaflet restriction versus coapting force: in vitro studies. Circulation 1997; 96: 1826-34.

15 Komeda M, Glasson J R, Bolger A F et al. - Threedimensional regional dynamics of the normal mitral annulus during left ventricular ejection. J Thorac Cardiovasc. Surg 1996; 111: 574-85.

16 Kunzelman K S, Cochran R P, Verrier E D, Eberhart R $\mathrm{C}$ - Anatomic basis for mitral valve modelling. $J$ Heart Valve Dis 1994; 3: 491-6.

17 Choo S J, Olomon J, Bowles C et al. - An in vitro study of the correlation between aortic valve diameter and mitral intertrigonal distance: a simple method to select the correct mitral annuloplasty ring size. $J$ Heart Valve Dis 1998; 7: 593-7.

18 Brock R C - The surgical and pathological anatomy of the mitral valve. Br Heart J 1952; 14: 489-513.

19 Cosgrove D M 3rd, Arcidi J M, Rodriguez L, Stewart W J, Powell K, Thomas J D - Initial experience with the Cosgrove-Edwards Annuloplasty System. Ann Thorac Surg 1995; 60: 499-504.

20 Camilleri L, Filaire M, Repossini A et al. - Mitral annuloplasty with a flexible linear reducer. $J$ Card Surg 1995; 10: 99-103.
21 Salati M, Scrofani R, Santoli C - Posterior pericardial annuloplasty: a physicological correction? Eur J Cardiothorac Surg 1991; 5: 226-9.

22 Carpentier A - La valvuloplastie reconstitutive. Une douvelle technique de valvuloplastie mitrale. Presse Med 1969; 7: 251-3.

23 Duran C G \& Ubago J L - Clinical and hemodynamic performance of a totally flexible prosthetic ring for atrioventricular valve reconstruction. Ann Thorac Surg 1976; 22: 458-63.

24 Kono T, Sabbah H N, Stein P D, Brymer J F, Khaja F - Left ventricular shape as a determinant of functional mitral regurgitation in patients with severe heart failure secondary to either coronary artery disease or idiopathic dilated cardiomyopathy. J Am Cardiol 1991; 68: 355-9.

25 Kono T, Sabbah H N, Rosman H, Alam M, Jafri S, Goldstein S - Left ventricular shape is the primary determinant of functional mitral regurgitation in heart failure. J Am Coll Cardiol 1992; 20: 1594-8.

26 Carpentier A, Deloche A, Dauptain J et al. - A new reconstructive operation for correction of mitral and tricuspid insufficiency. $J$ Thorac Cardiovasc Surg 1971; 61: 1-13.

27 Okada Y, Shomura T, Yamaura Y, Yoshikawa J Comparison of the Carpentier and Duran prosthetic rings used in mitral reconstruction. Ann Thorac Surg 1995; 59: 658-63.

28 Pellegrini A, Quaini E, Colombo T, Lanfranchi M, Russo C, Vitali E - Posterior annuloplasty in the surgical treatment of mitral insufficiency. $J$ Heart Valve Dis 1993; 2: 633-8. 